\title{
A feasibility study characterising tumour microenvironment of longitudinally collected biopsies of pancreatic tumours upon endoscopic ultrasound guided radiofrequency ablation
}

\section{Patrick V. Lawrence}

Division of Molecular Pathology, Institute of Cancer Research (ICR), London, UK

\section{Krisha Desai}

Division of Molecular Pathology, Institute of Cancer Research (ICR), London, UK

Christopher Wadsworth

Imperial College, London, UK

\section{Nagina Mangal}

Imperial College, London, UK

\section{Nagy Habib}

Imperial College, London, UK

Anguraj Sadanandam ( $\sim$ anguraj.sadanandam@icr.ac.uk)

Division of Molecular Pathology, Institute of Cancer Research (ICR), London, UK https://orcid.org/00000001-8485-5150

Mikael Sodergren ( $\nabla$ m.sodergren@imperial.ac.uk)

Imperial College, London, UK

\section{Research Article}

Keywords: Radiofrequency ablation, EUS-RFA, FNAB, PDAC, tumour microenvironment, immunotherapy, tumour microenvironment, cancer subtypes, CAF subtypes, cancer-associated fibroblast, immune checkpoint genes

Posted Date: June 30th, 2021

DOl: https://doi.org/10.21203/rs.3.rs-668215/v1

License: (9) This work is licensed under a Creative Commons Attribution 4.0 International License. Read Full License 
1 A feasibility study characterising tumour microenvironment of longitudinally collected biopsies of pancreatic tumours upon endoscopic ultrasound guided radiofrequency

ablation

4 Patrick V. Lawrence ${ }^{1, \&}$, Krisha Desai ${ }^{1, \&}$, Christopher Wadsworth ${ }^{2}$, Nagina Mangal ${ }^{1,2}$, Nagy

5 Habib $^{2}$, Anguraj Sadanandam ${ }^{1,3, *}$, Mikael H. Sodergren ${ }^{2, *}$

$6 \quad{ }^{1}$ Systems and Precision Cancer Medicine Team, Division of Molecular Pathology, The Institute 7 of Cancer Research, London, UK

$8 \quad 2$ Department of Surgery and Cancer, Imperial College London, UK

$9{ }^{3}$ Centre for Global Oncology, Division of Molecular Pathology, The Institute of Cancer

10 Research, London, UK

$11{ }^{*}$ Co-corresponding authors

$12{ }^{\&}$ Contributed equally to the work

\section{Highlights}

14 - First EUS-RFA clinical study to longitudinally collect fine-needle aspiration biopsy 15 (FNAB) and profile PDAC microenvironmental transcriptome changes

16 - The immuno-modulatory role, including change in checkpoint genes, of RFA-treated 17 samples depends on baseline FNAB molecular features

- Established FNAB-derived CAF culture and bulk tumour profiles demonstrated stromal and

19 cancer subtype remodelling in post-treated samples

20 - Classical PDA subtype genes are downregulated, and other subtype genes have no change 21 upon RFA treatment

- RFA post-chemotherapy offers a potential avenue for personalised combination anticheckpoint immunotherapy strategy 


\section{Abstract}

27 Most patients with pancreatic ductal adenocarcinoma (PDAC) are metastatic at presentation

28 with dismal prognosis warranting improved systemic therapy options. Longitudinal sampling

29 for assessment of treatment response poses a challenge for validating novel therapies. In this

30 feasibility study, we evaluate the role of endoscopic ultrasound (EUS)-guided serial fine-needle

31 aspiration biopsies (FNABs) to study the molecular changes associated with radiofrequency

32 ablation (RFA).

\section{Methods}

34 Two stage III inoperable gemcitabine-treated PDAC patients were recruited in the treatment 35 arm of ARDEO (ethically approved Phase-II prospective randomized clinical study). Post 36 examination, targeted RFA was delivered thrice, and sequential FNABs of tumour were taken 37 before and after treatment and analysed using a custom NanoString panel (144 genes) 38 consisting of cancer and cancer-associated fibroblast (CAFs) subtypes and immune changes. 39 CAF culture was established from one FNAB and characterised by immunofluorescence and 40 immunoblotting.

\section{Results}

42 Serial EUS-RFA treatments were well tolerated with no complications. Both patients had stable 43 disease immediately after EUS-RFA, however, with different survival outcomes. Two-course $44 \quad$ RFA led to upregulation of $C D 1 E$ gene (involved in antigen presentation) in both, patient 1 and 2 (4.5 and 3.9-fold) compared to baseline. Patient 1 showed increased T cell genes (CD4 46 -8.7-fold, $C D 8$-35.7-fold), cytolytic function (6.4-fold) and inflammatory response (8-fold).

47 Greater than 2-fold upregulation of immune checkpoint genes was observed post $2^{\text {nd }}$ RFA in 48 patient 1, patient 2 or both. Further, two-course RFA led to increased PDGFRa (4.5-fold) and 49 CAF subtypes B and C genes in patient 1 and subtypes A, B and D genes in patient 2. Patient250 derived CAFs post $1^{\text {st }}$ RFA showed expression of PDGFR $\alpha$, POSTN and MYH11 proteins. 51 Finally, RFA led to downregulation of classical PDA subtype in both patients. 
55 For the first time, this feasibility study validates longitudinal sampling by EUS-FNABs as an 56 appropriate research tool to study tumour microenvironmental changes associated with local

57 PDAC immunomodulatory treatments like RFA.

\section{Keywords}

59 EUS-RFA, FNAB, PDAC, tumour microenvironment, immunotherapy, tumour 60 microenvironment, cancer subtypes, CAF subtypes, cancer-associated fibroblast, immune 61 checkpoint genes.

\section{Introduction}

65 Pancreatic ductal adenocarcinoma (PDAC) is the third most common cause of cancer death after colorectal and lung cancer(Ansari et al. 2016), with a five-year survival rate of $<5 \%$ despite significant advances in cancer management for other solid organ malignancies over the last few decades. At presentation, only $5-25 \%$ of patients are eligible for radical curative surgery and, even in these patients, only $30 \%$ of patients will survive five years. At diagnosis, $\sim 40 \%$ of patients have locally advanced, unresectable, stage 3 disease, usually due to significant encasement of the superior mesenteric vein/portal vein or involvement of the superior mesenteric or hepatic arteries. The prognosis for this group of patients is extremely poor, with a median survival of 12-14 months on chemotherapy. While a small proportion of these patients respond well to chemotherapy and can subsequently undergo surgery, palliative chemotherapy remains the only treatment option in most patients(Gillen et al. 2010).

Radiofrequency ablation (RFA) describes destruction of the tumour using heat generated by a high frequency alternating current applied through an electrode tip. The tissue is heated to $60^{\circ} \mathrm{C}$, resulting in coagulative tissue necrosis at the center of the ablation zone. RFA is used to treat a few solid organ malignancies and is routinely used in both primary and secondary hepatocellular carcinoma (HCC)(Livraghi et al. 2000; M Pai et al. 2012; Madhava Pai et al.

81 2015; Weber et al. 2002). However, complete eradication of stage 3 PDAC is not usually 82 possible with RFA due to tumour proximity to major vascular structures and the duodenum 83 and a consequent risk of injury. Nevertheless, RFA of inoperable stage 3 PDAC through 84 laparotomy has been found to be safe. Indeed, a recent large series examining the procedure 
reported a median overall survival (OS) and disease-specific survival (DSS) of 20 and 23 months, respectively, with an RFA-related morbidity of $15 \%$ and overall mortality of $3 \%$ (Girelli et al. 2013), which compares to a median survival of 12-14 months on standard chemotherapy(Gillen et al. 2010).

Following RFA, the localised necrosis of the tumour initiates a cascade of events including release of proinflammatory signals, release of cellular debris representing a source of tumour antigens, and a host adaptive immune response against the tumour(Evrard et al. 2007). RFA appears to induce both innate and adaptive immune responses against the tumour through effective infiltration of dendritic cells, boosted antigen presentation, and an intensified $\mathrm{T}$ cell response(den Brok et al. 2006; Dromi et al. 2009; Haen et al. 2011; Napoletano et al. 2008; Zerbini et al. 2006). Recent studies have suggested that cancer-associated fibroblasts (CAFs), which are central components of the desmoplastic stroma found in most PDACs, can positively or negatively impact anti-tumour immune responses(Biffi et al. 2019; Kieffer et al. 2020; Neuzillet et al. 2019; Sahai et al. 2020). Others and we have recently reported heterogeneity in CAFs and identified different subtypes (subtypes A-B or inflammatory (i)CAF/myofibroblast (my)CAF/antigen presenting (ap)CAF) with distinct associations with immune cells(Elyada et al. 2019; Neuzillet et al. 2019). Moreover, cancer subtypes (classical, quasi-mesenchymal (QM) and exocrine-like) and similar subtypes of PDAC have been shown to be associated with immune/stromal changes and patient prognosis(Bailey et al. 2016; Collisson et al. 2011; Moffitt et al. 2015). Thus, a comprehensive understanding of the cell types within the tumour microenvironment (TME) is needed when assessing anti-tumour responses associated with RFA in which the immune system plays a critical modulatory role.

There have been no previous reports of longitudinal sampling of pancreatic tumours when treated with radiofrequency ablation in PDAC patients, which is necessary to understand the mechanism of action of the treatment. It is also unknown whether minimally invasive tissue sampling through endoscopic ultrasound (EUS)-guided fine needle aspiration biopsy (FNAB) is adequate for sampling tissues subjected to RFA for cellular and molecular analyses. This proof-of-concept study therefore aimed to determine the feasibility of studying the potential mechanism of action of RFA in pancreatic cancer through serial EUS-FNAB. 


\section{Study Design}

118 Patients were recruited through the treatment arm of the pilot phase of the ARDEO trial (A

119 phase II prospective Randomised clinical study of enDoscopic ultrasound guided 120 radiofrEquency ablation for inOperable pancreatic ductal adenocarcinoma). This is a 121 prospective randomised clinical study (REC reference: 18/SW/0103) in patients with stage 3 122 inoperable adenocarcinoma of the pancreas starting gemcitabine chemotherapy with a 1:1 randomisation. Patients in the treatment group receive up to 3 endoscopic ultrasound radiofrequency ablation procedures, each a month apart and sequential EUS FNABs were taken 125 during each procedure prior to RFA treatment. Written informed consent was taken from the 126 patients before enrolling into the study. Patients in the control group will receive best medical 127 care. A study flow diagram is illustrated in Fig 1a. PDAC patients in the treatment group were 128 recruited over 2 months at the Hammersmith Hospital, Du Cane Road, London.

Endoscopic-ultrasound guided radiofrequency ablation

130 Following EUS examination of the pancreas and primary tumour site, an FNAB of the tumour was taken and then targeted RF delivered using 10 Watts for 2 minutes per application using the Habib EUS RFA (Boston Scientific, MA, USA). For the second and third EUS examinations, the ablation zone is examined, and a fine needle biopsy taken prior to RFA.

134 FNABs were snap frozen in liquid nitrogen and transported on dry ice. Routine biochemistry tests including bilirubin and CA19-9 were analysed at Imperial College Healthcare NHS Trust laboratory.

\section{Nucleic acid extractions from FNABs}

138 Flash frozen FNABs were homogenised in Precellys beaded tubes (Bertin Technologies ${ }^{\mathrm{TM}}$, 139 Montigny-le-Bretonneux, France) containing $700 \mu \mathrm{l}$ of lysis buffer using a tissue homogeniser 140 (Bertin Technologies ${ }^{\mathrm{TM}}$, Montigny-le-Bretonneux, France). RNA from tumour lysates was 141 extracted using AllPrep DNA/RNA/miRNA Universal Kit (Qiagen ${ }^{\mathrm{TM}}$, Manchester, UK) 142 according to manufacturer's instructions. RNA yield was estimated by Nanodrop 2000 143 (Thermo Fisher Scientific ${ }^{\mathrm{TM}}$, Delaware, USA). 
$147100 \mathrm{ng}$ of total mRNA was used to run a custom designed human gene panel encompassing 148 cancer, immune and cancer associated fibroblast genes along with house-keeping reference 149 genes for data normalization (144 genes, Table S1). Tumour samples were run on the 150 nCounter ${ }^{\circledR}$ Max analysis system (NanoString Technolgies, CA, USA) as per the protocol 151 previously published by us(Ragulan et al. 2019). Data was assessed for quality, normalised to

152 housekeeping genes and $\log _{2}$ transformed using nSolver 4.0 software. Gene expression values 153 were plotted using GraphPad Prism Version 8.0 (for Windows, GraphPad Software; La Jolla,

154 CA, USA, www.graphpad.com).

155

156

\section{Gene Scores and subtypes}

Gene scores for cancer subtypes, T cell, B cell, cytolytic function and inflammation were obtained by averaging the expression of genes specific to the respective subtype, cell types or immune function(Forero et al. 2019; Fulkerson et al. 2004; Newman et al. 2015; Szabo et al. 2019; Weng, Araki, and Subedi 2012; Zheng et al. 2017). List of subtype and cell type specific genes used here to arrive at scores can be found in Table S2. CAF subtype scores were derived by averaging the expression of top differentially expressed genes representing pCAF subtypes(Neuzillet et al. 2019) for each sample. PDA subtypes(Collisson et al. 2011) were predicted by the nearest template prediction method (NTP)(Hoshida 2010) using subtype specific genes identified previously(Collisson et al. 2011).

\section{Isolation of patient-derived cancer-associated fibroblasts from EUS-FNABS}

Cancer associated fibroblasts from fresh FNABs were isolated by outgrowth method (Apte et al. 1998; Bachem et al. 1998). Briefly, tissue biopsies were transported in advanced Dulbecco's Modified Eagle Medium/Ham's F12 (Gibco, NY, USA) containing 10\% fetal bovine serum (FBS, Qualified HI; Gibco, NY, USA) and 1\% penicillin-streptomycin solution (Gibco, NY, USA) (complete media). FNABs were washed twice in phosphate buffered saline (PBS) containing $1 \%$ penicillin-streptomycin solution and cut into smaller pieces using sterile scalpels. Tissue digestion was performed in PBS containing $1 \mathrm{mM}$ ethylenediamine-tetraacetic acid (PBS-EDTA) along with 2x TrypLE (Gibco, NY, USA) and $20 \mu$ of DNase enzyme for $1 \mathrm{~h}$ at $37^{\circ} \mathrm{C}$. Following digestion, tubes were centrifuged for $5 \mathrm{~min}$ at $1200 \mathrm{rpm}$ and the tissue pellets were resuspended in $2 \mathrm{ml}$ of ACK lysis buffer (Gibco, NY, USA) to deplete red blood cells by incubating on ice for $3 \mathrm{~min} .5 \mathrm{ml}$ of complete media was added to neutralize the 
177 solution and tubes were centrifuged for $5 \mathrm{~min}$ at $1200 \mathrm{rpm} .3 \mathrm{ml}$ of pre-warmed complete media

178 was added to the tissue pellet and contents were placed on $35 \mathrm{~mm}$ dishes. After a week, 179 fibroblasts that grew out from the tissue bits were trypsinised and passaged once a week. Cells 180 were maintained at $37^{\circ} \mathrm{C}$ in a $5 \% \mathrm{CO}_{2}$ incubator. Cells were screened for mycoplasma by polymerase chain reaction and found to be negative (Figure S1). DNA for STR profiling was sent to Eurofins Genomics (Wolverhampton, UK).

\section{Immunofluorescence}

$2 \times 10^{4}$ patient-derived CAFs were seeded on 8-well chambered coverslips (ibidi, Thistle Scientific, Glasgow, UK) in complete growth media. Next day, media was aspirated and cells were washed twice with cold PBS and fixed in 4\% paraformaldehyde for $15 \mathrm{~min}$ at room temperature (RT). After two gentle washes in PBS, cell permeabilisation and blocking was performed using PBS containing $5 \%$ bovine serum albumin (BSA) $+0.3 \%$ Triton-X-100 for 1 $h$ at RT. Cells were incubated with primary antibodies overnight at $4^{\circ} \mathrm{C}$. On the following day, cells were washed thrice with PBS and incubated with secondary antibody (in PBS containing $1 \% \mathrm{BSA}+0.05 \%$ Tween-20) for $1 \mathrm{~h}$ at RT in a moist dark chamber. Cells were washed thrice with PBS and stained with 4',6-diamidino-2-phenylindole (DAPI) (1:1000 in PBS) for $10 \mathrm{~min}$ at RT. Coverslips were mounted on $50 \mu \mathrm{l}$ of VectaShield mounting media (Vector Labs, Detroit, USA) and imaged using Zeiss LSM700 (Jena, Germany). Antibody information is listed in Table S3.

\section{Immunoblotting}

$0.6 \times 10^{6}$ patient derived CAFs were seeded in complete growth media in $60 \mathrm{~mm}$ dishes. Next day, media was aspirated, and cells were washed twice with cold PBS. $30 \mu \mathrm{l}$ of cold NP40 lysis buffer (50mM Tris-HCL, 150mM sodium chloride, 1\% NP40) containing phosphatase and protease inhibitors (Pierce, Life Technologies Corporation, CA, USA) was added to obtain protein lysates. Protein concentrations were determined using BCA method (Pierce, Life Technologies Corporation, CA, USA) and $10 \mu \mathrm{g}$ of protein was fractionated onto $12 \%$ sodium dodecyl sulphate-polyacrylamide gels. Blotting was performed on polyvinylidene difluoride (PVDF) membranes for $1 \mathrm{~h}$ at $100 \mathrm{~V}$. Membranes were blocked in PBS containing 5\% BSA + $0.12 \%$ Tween-20 for $1 \mathrm{~h}$ followed by probing with primary antibodies, overnight at $4{ }^{\circ} \mathrm{C}$. Next day, membranes were washed and incubated with anti-mouse/rabbit secondary antibodies conjugated to horseradish peroxidase. Immunoreactive bands were visualised on Licor 
208 Odyssey Fc (Cambridge, UK) using enhanced chemiluminescence (ECL, Merck Millipore,

209 Darmstadt, Germany). $\beta$ actin was used a loading control. Antibody information is listed in 210 Table S3.

211 Statistics

212 To assess RFA-mediated transcriptome modulation in serial FNABs, $\log _{2}$ transformed gene 213 expression values at T2 (after $1^{\text {st }}$ RFA) and T3 (after $2^{\text {nd }}$ RFA) were subtracted from T1 214 (baseline) and denoted as difference. $2^{\wedge}$ (difference) was then performed to calculate fold 215 change in gene expression over the course of RFA treatment.

\section{Results}

219 The first two patients recruited in the treatment arm of the ARDEO trial were included in this 220 feasibility study. All EUS-RFA procedures were uneventful with no observed clinical 221 complications.

Patient 1

223 Patient 1 was a 71-year-old female with no significant co-morbidities who presented with 224 obstructive jaundice and weight loss in November 2018. On presentation she had a bilirubin of 225223 and cancer antigen (CA)19-9 of 503 and underwent endoscopic retrograde 226 cholangiopancreatography (ERCP) with metal stent insertion and EUS and biopsy of a mass in 227 the neck of the pancreas in early December 2018. The tumour was found to be an 228 adenocarcinoma involving the splanchnic vasculature and deemed inoperable with a 229 radiological staging of tumour $(\mathrm{T})$ and nodal status $(\mathrm{N})$ of T4N1. She was enrolled in the 230 ARDEO trial on $24^{\text {th }}$ January 2019 and started gemcitabine chemotherapy that day. She 231 underwent EUS with RFA ablations on $13^{\text {th }} \mathrm{Feb}, 13^{\text {th }}$ March and $17^{\text {th }}$ April. There were no 232 complications or morbidity relating to the EUS or RFA treatment. However, she did suffer with 233 Grade 1 nausea related to gemcitabine treatment that was fully responsive to the anti-nausea 234 drug, domperidone. The patient elected to take a treatment break after cycle 6 of gemcitabine 235 as she was thinking of relocating and wanted to go away on holiday. At the time of stopping treatment, she had stable disease radiologically and her CA19-9 had decreased from 4887 when 
237 enrolled in the trial to 392 on the $25^{\text {th }}$ of April, which was after her final EUS treatment (cycle

238 3). After cycle 3, the CA19-9 increased to 1324 at the end of cycle 6 and at the start of the 239 treatment break. She re-presented in October with liver metastases, portal vein thrombosis and 240 a CA19-9 of 503,293 and underwent one cycle of CAPOX (oxaliplatin and capecitabine)

241 chemotherapy but sadly passed away on the $15^{\text {th }}$ of November.

\section{Patient 2}

243 Patient 2 is a 62-year-old male with a background of Type 2 Diabetes Mellitus, hypertension 244 and hypercholesterolaemia who presented in December 2018 with abdominal pain, weight loss 245 and jaundice. He never had elevated tumour markers (CA-19-9) since diagnosis. On cross246 sectional imaging, he was found to have mass in the neck/body of the pancreas which was 247 invading the splanchnic vessels with EUS biopsy confirming a diagnosis of adenocarcinoma 248 with a radiological staging of T4N2M0 (M - represents presence of metastasis). He was 249 enrolled in the ARDEO study on $2^{\text {nd }}$ of February 2019 and started first cycle of Gemcitabine 250 on 4 days later. He underwent EUS, biopsy and then RFA on $6^{\text {th }}$ of March, $3^{\text {rd }}$ April and $10^{\text {th }}$ 251 of May. He subsequently completed 10 cycles of gemcitabine maintaining stable disease on cross-sectional imaging and then started chemo-radiotherapy (capecitabine and 20 Fractions) in February which was stopped in March 2020 due to the risk of coronavirus at the height of the pandemic in London. Fig. 1b shows representative ultrasound images of tumour at baseline, tumour and ablation site after 3 courses of EUS-RFA and 16 months post treatment of patient 2S. Updated imaging on $28^{\text {th }}$ of August 2020 shows stable disease (Fig. 1b).

Feasibility of evaluating whether EUS-RFA treatment elicits an anti-tumour immune response in PDAC

259 To obtain insight into RFA mediated modulation of the TME, we performed transcriptomic 260 profiling of FNABs collected longitudinally over three courses of RFA for the two patients enrolled in our proof-of-principle study. A schematic representation of sample collection and approaches is outlined in Fig. 2a. A low-cost custom NanoString panel was designed to include genes for PDA and CAF subtypes identified previously (Collisson et al. 2011; Neuzillet et al. 2019), along with markers to assess changes in the TME as a result of RFA treatment (Table S2). Initial unsupervised hierarchical clustering of the six tumour samples grouped T3 FNABs (after $2^{\text {nd }}$ RFA) of both the patients together and showed enrichment of T cell markers, immune checkpoint genes and increased fibrosis observed as upregulated CAF markers. T3 samples were most similar to T2 (after $1^{\text {st }}$ RFA) profile but only in patient 2. T1 (baseline) and T2 (after 
$1^{\text {st }}$ RFA) from patient 1 clustered together and shared similar expression profiles to $\mathrm{T} 1$

270 (baseline) from patient 2, except the latter was highly enriched for $\mathrm{T}$ and $\mathrm{B}$ cell genes along

271 with cytolytic function markers, Fig. 2b. This suggests that the baseline sample from patient 2

272 was already immune-enriched, which is not the case with patient 1.

273 We first assessed RFA-induced immunological changes resulting in an increased adaptive 274 immune infiltration into the tumours. A high T (7.3-fold) and B (2.2-fold) cell score at T3 in 275 patient 1 compared to baseline and T2 was observed. There was no change in $\mathrm{T}$ and $\mathrm{B}$ cell 276 score in patient 2 at T2 and T3 compared to baseline (Fig. 2c; shows $\log _{2}$ expression). Overall, 277 the T and B cell changes vary in individual patients after RFA treatment. Ratio of cytotoxic $278 \mathrm{CD}^{+} \mathrm{T}$ cells by $\mathrm{FOXP}^{+}$regulatory $\mathrm{T}$ cells has been shown to have prognostic and predictive 279 value in multiple cancer types(Asano et al. 2016; Goto et al. 2018; Suzuki et al. 2010). We 280 assessed this ratio using CD8A and FOXP3 gene expression and found greater than 3-fold 281 upregulation in patient 1 at both T2 and T3 compared to baseline. This ratio, however, was 282 lower in patient 2 after RFA treatment (Fig. 2d).

283 Further, genes associated with proteins that participate in antigen presentation to $\mathrm{T}$ cells via 284 major histocompatibility complexes I and II (CDIE for MHC-I(Angenieux et al. 2000; 285 Blumberg et al. 1995) and HLADQA1 for MHC-II(Majumder, Gomez, and Boss 2006) were 286 also highly upregulated upon RFA. There was a 4.5-fold and 5.3-fold increase in expression of $287 C D 1 E$ and $H L A D Q A 1$ respectively at $\mathrm{T} 3$ time point in patient $1 . C D 1 E$ expression in patient 2 288 at T3 was also found to be 3.9-fold greater than at baseline but a decreasing trend in HLADQA1 289 was observed (Fig. 2e). However, we warrant further validation of MHC-I and -II using 290 additional gene sets representing the complexes in the future.

291 Next, we investigated the different immune cell types that may have been recruited to the tumours because of RFA. Patient 1 showed RFA course-dependent upregulation in T cell markers - CD4 (8.7-fold at T3) and CD8 (35.7-fold at T3), along with a 3.7-fold increase in markers representing NK cells. Genes representing macrophages and mast cells showed less than 2-fold change in patient 1 with respect to RFA treatment. CD4 expression and CD8 expression showed 2-fold and 3.2-fold decrease at T3 compared to baseline in patient 2. The baseline levels of CD4 and CD8 were, however, higher in patient 2 compared to patient 1, suggesting that patient 2 was already inflamed with CD4 and CD8 T cells before RFA. 
299 A reduction in transcript levels of genes representing macrophages (2.6-fold) and mast cells 300 (3.7-fold) and no change in NK cell genes was observed in patient 2 post-RFA (at T3) 301 compared to baseline (Fig. 2f). Further, apart from upregulated T cell markers, a 6.4-fold and 3028 -fold increase in cytotoxic T cell function score and inflammation score, respectively was 303 observed at T3 compared to baseline in patient 1. Decrease in cytolytic function score (14.17304 fold at T3) and a 2-fold increase in inflammation score was observed after 2 courses of RFA 305 in patient 2 (Fig. $2 \mathrm{~g}$ ).

306

307

308

309

310

311

312

313

314

315

316

317

318

319

320

321

322

323

324

325

326

327

328

329

330

$\mathrm{T}$ cell infiltration after RFA treatment has been shown to be accompanied by an exhausted $\mathrm{T}$ cell phenotype marked by increased expression of immune checkpoint genes(Fei et al. 2020). We studied the expression of known immune checkpoint genes with agonists/antagonists currently in clinical trials or approved for clinical use in other cancers (Hodi et al. 2018; Larkin et al. 2019; Overman et al. 2017). After 2 courses of RFA (T3), patient 1 showed $>2$-fold increase in CD274 (PDL1), IDO1, TNFRSF18 (GITR), Fig. 2h. Also, a >2-fold upregulation in PDCD1 (PD1), IDO1, TNFSF18 (GITRL) and TNFRSF18 (GITR) was observed in patient 2 at T3, shown in Fig. 2i. These suggests that both these patients may be amenable to anticheckpoint treatment after 2 courses of RFA treatment.

\section{Feasibility of evaluating whether RFA remodels the tumour stroma in PDAC}

Recent studies have reported loco-regional coagulation and necrosis as a result of RFA resulting in remodelling of the TME(Evrard et al. 2007; Livraghi et al. 2000). We investigated if the molecular changes brought about by RFA reflected a switch in our previously identified pCAFassigner (pCAF; cancer-associated fibroblast) subtypes(Neuzillet et al. 2019). Data from both patients at T3 indicated a marked upregulation (4.5-fold; patient 1 and 8.5-fold; patient 2 respectively) of pan-CAF marker - PDGFR $\alpha$. Patient 1 tumour also showed a 3.8-fold increase in ACTA2 ( $\alpha S M A)$ after 2 courses of RFA, Fig. 3a. RFA led to a modest increase in subtype B and $\mathrm{C}$ CAFs in patient 1, Fig. 3b. This is supported by our previous finding suggesting enhanced immune cell responses in pCAF subtype C-dominated tumours(Neuzillet et al. 2019), similar to inflammatory CAF subtype identified by Ohlund et al(Öhlund et al. 2017). In contrast, RFA treated patient 2 tumour (T3) showed a 3.6-fold, 9-fold and 5-fold upregulation of pCAF subtypes A, B and D, respectively (Fig. 3c). We then selected the genes that have been previously validated by immunohistochemistry to exclusively represent pCAF subtypes and found an enrichment of POSTN (subtype A), MYH11 (subtype B) and PDPN (subtype C) in both patients after RFA, however, the increase was pronounced particularly in patient 2 
tumour at T2 and T3, Fig. 3d. In order to validate CAF-specific expression of these markers,

332 we established cultures from patient FNABs and successfully obtained CAFs from patient 2

333 tumour at T2. A strong expression of pan-CAF markers - PDGFR $\alpha, \alpha$ SMA and VIM was

334 observed along with expression of subtype A (POSTN) and subtype B (MYH11) in the cultured

335 CAFs (Fig. 3e).

336 Feasibility of evaluating whether RFA remodels the cancer subtypes of PDAC

337 In order to study how the crosstalk between immune cells and CAFs in the TME regulates

338 cancer cells, we performed PDA subtyping of tumours pre- and post-RFA by NTP using 339 subtype specific genes described previously(Collisson et al. 2011). Our data indicated that both 340 patients at baseline were categorised into the classical PDA subtype. RFA led to a marked 341 decrease in genes representing classical PDA subtype in both patients at T3 compared to 342 baseline and T2, Fig. $3 \mathrm{f}$ and $3 \mathrm{~g}$. No particular change in exocrine-like or quasi-mesenchymal 343 PDA subtype was observed in both the patients upon RFA. Overall, the concomitant 344 enrichment of adaptive immune response and immune promoting CAF subtype suggest RFA mediated remodelling of stroma in PDAC.

\section{Discussion}

348 This feasibility study established the value of longitudinal sampling with EUS-FNABs to study 349 local TME changes occurring in response to RFA in two patients. We successfully isolated 350 RNA from these biopsies to comprehensively profile immune, CAF, and cancer cell gene 351 expression using a panel of genes associated with PDAC subtypes (both cancer and CAF) and 352 immune cell types. Using inferential statistics, we established different patterns of longitudinal 353 remodeling of immune cells and stroma after RFA in these two patients, enrolled in the 354 ARDEO trial, were treated with RFA, and who differed in terms of survival (not necessarily 355 associated with RFA), treatment response, and change in CA19-9 and bilirubin levels. While 356 both patients had stable disease after RFA treatment, one patient died due to other reasons. 357 This study clearly elucidates heterogeneity in response to RFA in different patients depending 358 on their baseline immune repertoire. This study paves the way to access samples longitudinally 359 during treatment in clinical trials with EUS-RFA (to our knowledge for the first time) to assess 360 the molecular changes and subtypes associated with responses to therapy. 
361 We detected changes in genes participating in antigen presentation and $\mathrm{T}$ and $\mathrm{B}$ cell scores in 362 response to RFA in one patient. Similar results have been reported in a rat model of HCC, 363 which showed that necrotic cells release factors for several hours to days after RFA treatment 364 leading to activation and maturation of dendritic cells. This further stimulates $\mathrm{CD} 4^{+}$and $\mathrm{CD} 8^{+}$ 365 effector T cells directed against the antigen MHC class I-dominant, class I-subdominant, and 366 class II-dominant epitopes (Schueller et al. 2004). Indeed, in mice, similar MHC changes and $367 \mathrm{~T}$ cell responses were found in urothelial carcinomas partially treated with RFA. Furthermore, 368 animals with eradication of the primary tumour resisted further challenge with tumour implants, implying a durable immunological memory response(Dromi et al. 2009).

370 Our observation of increased cytolytic function and inflammatory gene expression after RFA 371 in one patient corroborate with multiple studies in animals. For example, den Brok and 372 colleagues elegantly demonstrated in mice that a significant proportion of draining lymph 373 nodes contained tumour antigen following RFA than after vaccination. Furthermore, they 374 showed that a combination of in situ tumour ablation and checkpoint inhibitor (anti-CTLA-4; 375 depletion of regulatory $\mathrm{T}$ cells) therapy enhanced $\mathrm{CD} 8^{+} \mathrm{T}$ cell activation(den Brok et al. 2006). 376 Interestingly, in the neoadjuvant setting in a murine model, RFA treatment demonstrated 377 systemic immunity against cancer cells leading to better survival. This study suggests a 378 potential application of RFA in patients with tumour types associated with increased risk of 379 recurrence(Ito et al. 2015).

380 In a clinical trial of non-small cell lung cancer (NSCLC), Schneider and colleagues 381 demonstrated local and systemic increase in $\mathrm{CD}^{+}$and $\mathrm{CD}^{+} \mathrm{T}$ cells after neoadjuvant RFA 382 (followed by surgery) in patients. These increased $\mathrm{T}$ cells in resected tissues were noticed 383 around the boundary where RFA was applied. They concluded that treatment of NSCLC with neoadjuvant RFA develops necrosis and provides long-term immunity against the tumour by activating $\mathrm{T}$ and dendritic cells(Schneider et al. 2016).

386 Our observation of enrichment of immune checkpoint genes in post-RFA treated tumours is consistent with a study by Shi et al., who found that RFA treatment of metastatic colorectal cancer showed increased PD-L1 expression and T cells in tumour tissues in a retrospective case controlled study(Shi et al. 2016). This provides a mechanism by which tumours can evade immune response and a potential target for combination therapy. Indeed, combined RFA and a CTLA-4 inhibitor (tremelimumab) was effective in a phase 1 trial of HCC patients. Those patients with clinical benefit from combination treatment of RFA and tremelimumab showed 
enhanced CD8 ${ }^{+} \mathrm{T}$ cells(Duffy et al. 2017). Further, in a study of patients undergoing RFA for locally advanced PDAC, Giardino and colleagues showed longitudinally enhanced adaptive immunity associated with $\mathrm{CD}^{+}, \mathrm{CD}^{+}$, effector memory $\mathrm{T}$ cells and dendritic cells(Giardino et al. 2017), suggesting potential opportunity to target PDAC with a combination of RFA and immune checkpoint inhibitor(Shi et al. 2016).

398

Co-enrichment of cytotoxic immune infiltrates with pCAF subtypes B and C in post-RFA patient 1 FNAB confirms our previous finding, along with other studies, of immune cell enrichment in PDACs dominated by subtype B/C CAFs or iCAFs(Elyada et al. 2019; Helms, Onate, and Sherman 2020; Neuzillet et al. 2019). Successfully established patient-derived CAFs from limited FNAB material confirmed the expression of the pCAF subtype A and B marker, as found in the bulk tumour sample. Patient-derived cancer and CAF cultures from the extended ARDEO study cohort will further establish the consequences of RFA on this crosstalk with a view on developing combination treatment strategies.

406 Nevertheless, it is important to note that patient 2 had increased anti-tumour immune infiltrate 407 at baseline itself, potentially responsible for prolonged overall survival. RFA courses led to no 408 particular increase in $\mathrm{T}$ and $\mathrm{B}$ cells or cytolytic activity. Interestingly, immune checkpoint 409 genes were upregulated upon RFA in patient 2. The overall immune milieu supports that patient

4102 may have been a contender for immune checkpoint therapy. Further, response to RFA in the 411 two patients was found to be heterogeneous providing an opportunity to obtain deeper insights 412 into immune signaling in pre- and post-RFA samples. In addition, how these changes are 413 associated with prior treatment, like gemcitabine, may be addressed in the larger sample set 414 which is part of the ARDEO trial.

415 Both the patients at baseline showed enrichment of classical PDA subtype genes which were 416 dramatically downregulated over the RFA courses. However, there was no drastic switch in 417 the subtype to QM or exocrine-like, suggesting that RFA may be controlling cancer cells from 418 switching to poor prognostic cancer type.

419 The results from this feasibility study need to be validated in the larger ARDEO cohort and 420 other datasets of PDAC patients treated with RFA. Although these analyses suggest RFA421 mediated change in immune, CAF, and cancer cell gene signatures, the biological implication 422 of their presence and spatial distribution requires deeper investigation. Despite the limitations 423 of these data from two patients and only 144 genes, this is the first feasibility study to report 
424 transcriptomic profile of the TME during RFA treatment by longitudinal sampling using serial

425 EUS-FNABs in PDAC patients.

426

427

428

429 Acknowledgements

430 We thank Mrs. Chanthirika Ragulan (Institute of Cancer Research) for supporting with 431 nCounter analysis, and Joanna Nicholls, research manager at the department of surgery 432 (Imperial College of London) for logistical support.

\section{$433 \quad$ Funding}

434 Funding, in part, was secured from EMcision and badged by NIHR for conducting the trial. No 435 other funding source to be declared.

\section{Disclosures}

$437 \mathrm{NH}$ has acted as a previous founder of EMcision which has now been acquired by Boston 438 Scientific. AS received grants from Merck, Pierre Fabre and Bristol Myers Squibb and is an 439 inventor on patents on Colorectal cancer classification with differential prognosis and 440 personalized therapeutic responses (PCT/IB2013/060416); Prognostic and treatment response 441 prediction in gastric cancer - Priority Patent CSC/BP7295892; Patient classification and 442 prognostic method (GEP-NET) - Priority Patent - EP18425009.0; and Molecular predictors of 443 therapeutic response to specific anti-cancer agents (US9506926B2). AS received consultant 444 honoraria from Ploughshare Innovations and serves as an advisor in Diagnostring Laboratories 445 and 4baseCare. MHS has received/s consultancy honoraria from Verb Surgical and EMMAC 446 Life Sciences and receives a research grant from MiNA Therapeutics. PVL, KD, CW and NM 447 have no conflict of interest to declare. 
452

453

454

455

456

457

458

459

460

461

462

463

464

465

466

467

468

469

470

471

472

473

474

475

476

477

478

479

480

481

482

483

484

485

486

487

488

489

490

491

492

493

494

495

496

497

498

499
Angenieux, C et al. 2000. "Characterization of CD1e, a Third Type of CD1 Molecule Expressed in Dendritic Cells." The Journal of biological chemistry 275(48): 37757-64.

Ansari, Daniel et al. 2016. "Pancreatic Cancer: Yesterday, Today and Tomorrow." Future oncology (London, England) 12(16): 1929-46.

Apte, M V et al. 1998. "Periacinar Stellate Shaped Cells in Rat Pancreas: Identification, Isolation, and Culture." Gut 43(1): 128-33.

Asano, Y et al. 2016. "Tumour-Infiltrating CD8 to FOXP3 Lymphocyte Ratio in Predicting Treatment Responses to Neoadjuvant Chemotherapy of Aggressive Breast Cancer." The British journal of surgery 103(7): 845-54.

Bachem, M G et al. 1998. "Identification, Culture, and Characterization of Pancreatic Stellate Cells in Rats and Humans." Gastroenterology 115(2): 421-32.

Bailey, Peter et al. 2016. "Genomic Analyses Identify Molecular Subtypes of Pancreatic Cancer." Nature 531(7592): 47-52.

Biffi, Giulia et al. 2019. "IL1-Induced JAK/STAT Signaling Is Antagonized by TGF $\beta$ to Shape CAF Heterogeneity in Pancreatic Ductal Adenocarcinoma." Cancer discovery 9(2): 282301.

Blumberg, R S et al. 1995. "Structure and Function of the CD1 Family of MHC-like Cell Surface Proteins." Immunological reviews 147: 5-29.

den Brok, M H M G M et al. 2006. "Efficient Loading of Dendritic Cells Following Cryo and Radiofrequency Ablation in Combination with Immune Modulation Induces AntiTumour Immunity." British journal of cancer 95(7): 896-905.

Collisson, Eric A et al. 2011. "Subtypes of Pancreatic Ductal Adenocarcinoma and Their Differing Responses to Therapy." Nature medicine 17(4): 500-503.

Dromi, Sergio A et al. 2009. "Radiofrequency Ablation Induces Antigen-Presenting Cell Infiltration and Amplification of Weak Tumour-Induced Immunity." Radiology 251(1): 58-66.

Duffy, Austin G et al. 2017. "Tremelimumab in Combination with Ablation in Patients with Advanced Hepatocellular Carcinoma." Journal of hepatology 66(3): 545-51.

Elyada, Ela et al. 2019. "Cross-Species Single-Cell Analysis of Pancreatic Ductal Adenocarcinoma Reveals Antigen-Presenting Cancer-Associated Fibroblasts." Cancer discovery 9(8): 1102-23.

Evrard, Serge et al. 2007. "Cytokines Pattern after Surgical Radiofrequency Ablation of Liver Colorectal Metastases.” Gastroenterologie clinique et biologique 31(2): 141-45.

Fei, Qinglin et al. 2020. "High-Dimensional Single-Cell Analysis Delineates Radiofrequency Ablation Induced Immune Microenvironmental Remodeling in Pancreatic Cancer." Cell Death \& Disease 11(7): 589. https://doi.org/10.1038/s41419-020-02787-1.

Forero, Adriana et al. 2019. "Differential Activation of the Transcription Factor IRF1 Underlies the Distinct Immune Responses Elicited by Type I and Type III Interferons." Immunity 51(3): 451-464.e6.

Fulkerson, Patricia C et al. 2004. "Pulmonary Chemokine Expression Is Coordinately Regulated by STAT1, STAT6, and IFN-Gamma." Journal of immunology (Baltimore, Md. : 1950) 173(12): 7565-74.

Giardino, Alessandro et al. 2017. "Immunomodulation after Radiofrequency Ablation of Locally Advanced Pancreatic Cancer by Monitoring the Immune Response in 10 Patients." Pancreatology: official journal of the International Association of Pancreatology (IAP) ... [et al.] 17(6): 962-66.

Gillen, Sonja et al. 2010. "Preoperative/Neoadjuvant Therapy in Pancreatic Cancer: A Systematic Review and Meta-Analysis of Response and Resection Percentages." PLoS 
Girelli, Roberto et al. 2013. "Results of 100 Pancreatic Radiofrequency Ablations in the Context of a Multimodal Strategy for Stage III Ductal Adenocarcinoma." Langenbeck's archives of surgery 398(1): 63-69.

Goto, Wataru et al. 2018. "Predictive Value of Improvement in the Immune Tumour Microenvironment in Patients with Breast Cancer Treated with Neoadjuvant Chemotherapy." ESMO $\quad$ open $000305-\mathrm{e} 000305$. https://pubmed.ncbi.nlm.nih.gov/30233820.

Haen, Sebastian P et al. 2011. "More than Just Tumour Destruction: Immunomodulation by Thermal Ablation of Cancer." Clinical \& developmental immunology 2011: 160250.

Helms, Erin, M Kathrina Onate, and Mara H Sherman. 2020. "Fibroblast Heterogeneity in the Pancreatic Tumour Microenvironment." Cancer discovery 10(5): 648-56.

Hodi, Frank Stephen et al. 2018. "Nivolumab plus Ipilimumab or Nivolumab Alone versus Ipilimumab Alone in Advanced Melanoma (CheckMate 067): 4-Year Outcomes of a Multicentre, Randomised, Phase 3 Trial." The Lancet. Oncology 19(11): 1480-92.

Hoshida, Yujin. 2010. "Nearest Template Prediction: A Single-Sample-Based Flexible Class Prediction with Confidence Assessment." PloS one 5(11): e15543-e15543. https://pubmed.ncbi.nlm.nih.gov/21124904.

Ito, Fumito et al. 2015. "Immune Adjuvant Activity of Pre-Resectional Radiofrequency Ablation Protects against Local and Systemic Recurrence in Aggressive Murine Colorectal Cancer." PloS one 10(11): e0143370.

Kieffer, Yann et al. 2020. "Single-Cell Analysis Reveals Fibroblast Clusters Linked to Immunotherapy Resistance in Cancer." Cancer discovery 10(9): 1330-51.

Larkin, James et al. 2019. "Five-Year Survival with Combined Nivolumab and Ipilimumab in Advanced Melanoma." The New England journal of medicine 381(16): 1535-46.

Livraghi, T et al. 2000. "Hepatocellular Carcinoma: Radio-Frequency Ablation of Medium and Large Lesions.” Radiology 214(3): 761-68.

Majumder, Parimal, Jorge A Gomez, and Jeremy M Boss. 2006. "The Human Major Histocompatibility Complex Class II HLA-DRB1 and HLA-DQA1 Genes Are Separated by a CTCF-Binding Enhancer-Blocking Element." The Journal of biological chemistry 281(27): 18435-43.

Moffitt, Richard A et al. 2015. "Virtual Microdissection Identifies Distinct Tumour- and Stroma-Specific Subtypes of Pancreatic Ductal Adenocarcinoma." Nature genetics 47(10): 1168-78.

Napoletano, Chiara et al. 2008. "RFA Strongly Modulates the Immune System and AntiTumour Immune Responses in Metastatic Liver Patients." International journal of oncology 32(2): 481-90.

Neuzillet, Cindy et al. 2019. "Inter- and Intra-Tumoural Heterogeneity in Cancer-Associated Fibroblasts of Human Pancreatic Ductal Adenocarcinoma." The Journal of pathology 248(1): 51-65.

Newman, Aaron $\mathrm{M}$ et al. 2015. "Robust Enumeration of Cell Subsets from Tissue Expression Profiles." Nature methods 12(5): 453-57.

Öhlund, Daniel et al. 2017. "Distinct Populations of Inflammatory Fibroblasts and Myofibroblasts in Pancreatic Cancer." The Journal of experimental medicine 214(3): 579-96.

Overman, Michael J et al. 2017. "Nivolumab in Patients with Metastatic DNA Mismatch Repair-Deficient or Microsatellite Instability-High Colorectal Cancer (CheckMate 142): An Open-Label, Multicentre, Phase 2 Study." The Lancet. Oncology 18(9): 1182-91.

Pai, M et al. 2012. "Radiofrequency Assisted Liver Resection: Analysis of 604 Consecutive Cases." European journal of surgical oncology: the journal of the European Society of 
Pai, Madhava et al. 2015. "Endoscopic Ultrasound Guided Radiofrequency Ablation, for Pancreatic Cystic Neoplasms and Neuroendocrine Tumours." World journal of gastrointestinal surgery 7(4): 52-59. Stratify Colorectal Cancer into Molecular Subtypes." Scientific Reports 9(1): 7665. https://doi.org/10.1038/s41598-019-43492-0.

Sahai, Erik et al. 2020. "A Framework for Advancing Our Understanding of Cancer-Associated Fibroblasts." Nature reviews. Cancer 20(3): 174-86.

Schneider, Thomas et al. 2016. "Immune Response After Radiofrequency Ablation and Surgical Resection in Nonsmall Cell Lung Cancer." Seminars in thoracic and cardiovascular surgery 28(2): 585-92.

Schueller, Gerd et al. 2004. "Expression of Heat Shock Proteins in Human Hepatocellular Carcinoma after Radiofrequency Ablation in an Animal Model." Oncology reports 12(3): 495-99.

Shi, Liangrong et al. 2016. "PD-1 Blockade Boosts Radiofrequency Ablation-Elicited Adaptive Immune Responses against Tumour." Clinical cancer research : an official journal of the American Association for Cancer Research 22(5): 1173-84.

Suzuki, Hiroyuki et al. 2010. "Intratumoural CD8(+) T/FOXP3 (+) Cell Ratio Is a Predictive Marker for Survival in Patients with Colorectal Cancer." Cancer immunology, immunotherapy: CII 59(5): 653-61.

Szabo, Peter A et al. 2019. "Single-Cell Transcriptomics of Human T Cells Reveals Tissue and Activation Signatures in Health and Disease." Nature communications 10(1): 4706.

Weber, Jean-Christophe et al. 2002. "New Technique for Liver Resection Using Heat Coagulative Necrosis." Annals of surgery 236(5): 560-63.

Weng, Nan-ping, Yasuto Araki, and Kalpana Subedi. 2012. "The Molecular Basis of the Memory T Cell Response: Differential Gene Expression and Its Epigenetic Regulation.” Nature reviews. Immunology 12(4): 306-15.

Zerbini, Alessandro et al. 2006. "Radiofrequency Thermal Ablation of Hepatocellular Carcinoma Liver Nodules Can Activate and Enhance Tumour-Specific T-Cell Responses." Cancer research 66(2): 1139-46.

Zheng, Chunhong et al. 2017. "Landscape of Infiltrating T Cells in Liver Cancer Revealed by Single-Cell Sequencing." Cell 169(7): 1342-1356.e16. 
592 Figure 1. EUS-RFA in human PDAC. a. Schematic representation of ARDEO study cohort,

593 b. Representative ultrasound images of the tumour and ablation site at baseline and after RFA 594 treatment. SOC - Standard of Care; CT - Computerised Tomography; RECIST - Response 595 Evaluation Criteria in Solid Tumours; FNAB - Fine Needle Aspiration Biopsy.

596 Figure 2. RFA induces immunogenicity in PDAC patients. a. Schematic illustration of 597 longitudinal FNABs and RFA courses along with approaches used in this study, b. Heatmap 598 depicting distribution of genes across samples and grouped by unsupervised hierarchical 599 clustering. Scale bar indicates $\log 2$ normalised expression ranging between -1 and +1 . Colour 600 bars represent appropriate CAF and PDA subtype-specific colours, c. Change in T and B cell 601 scores at T1, T2 and T3 in patient 1 and 2 respectively, d. Ratio of CD8A/FOXP3 in patient 1 602 and 2. e. Change in expression of genes participating in antigen presentation via MHC-I and II 603 at T1, T2 and T3 in patient 1 and 2 respectively, f. Heatmap representing RFA-mediated 604 regulation of genes associated with different immune cell types; single scale bar for both 605 patients depicting $\log 2$ normalised expression, g. Tumour-immune response in pre- and post606 RFA treated FNABs measured as cytolytic function and inflammatory score, $h$. and $i$. 607 Upregulation of immune checkpoint genes as a result of RFA in patient 1 and 2 respectively. 608 \# represents greater than equal to two-fold change in gene expression between T1 and T3. 609 Colour key represents T1 in red, T2 in blue and T3 in cyan.

610 Figure 3. RFA remodels the TME. a. Dot plot showing upregulation of pan-CAF markers $611 P D G F R \alpha$ and $A C T A 2(\alpha S M A)$ as a response to RFA in patient 1 and 2 respectively, b. and c. 612 Heatmaps representing a switch in the CAF subtypes over the course of RFA in patient 1 and 6132 respectively; separate scale bars for the two patients depicting averaged $\log _{2}$ normalised 614 subtype-specific CAF gene expression, d. Heatmap representing change in IHC validated 615 exclusive pCAF subtype-specific genes over the course of RFA in patient 1 and 2, e. Brightfield 616 (5x magnification) and confocal images of immunofluorescence staining of different CAF 617 markers in patient 2 T2-derived CAFs. Confocal images taken at 20x magnification; scale bar 618 represents $50 \mu \mathrm{m}$, f and g. Heatmaps representing a switch in the PDA subtypes over the course 619 of RFA in patient 1 and 2 respectively; colour bars represent NTP-derived PDA subtype and 620 separate scale bars for the two patients depicting averaged $\log 2$ normalised gene expression 621 associated with each PDA subtype. 


\section{Figure 1}

a.

\section{ARDEO Trial}

Patients $>=18$ years with histologically or cytologically proven inoperable Stage 3 PDA about to start Gemcitabine treatment

\section{Consented \& Screened for study}

Patients starting $3 \times 28$ days of SOC chemotherapy (Gemcitabine)

TRANSLATIONAL RESEARCH

FNAB and blood collection for research

b.

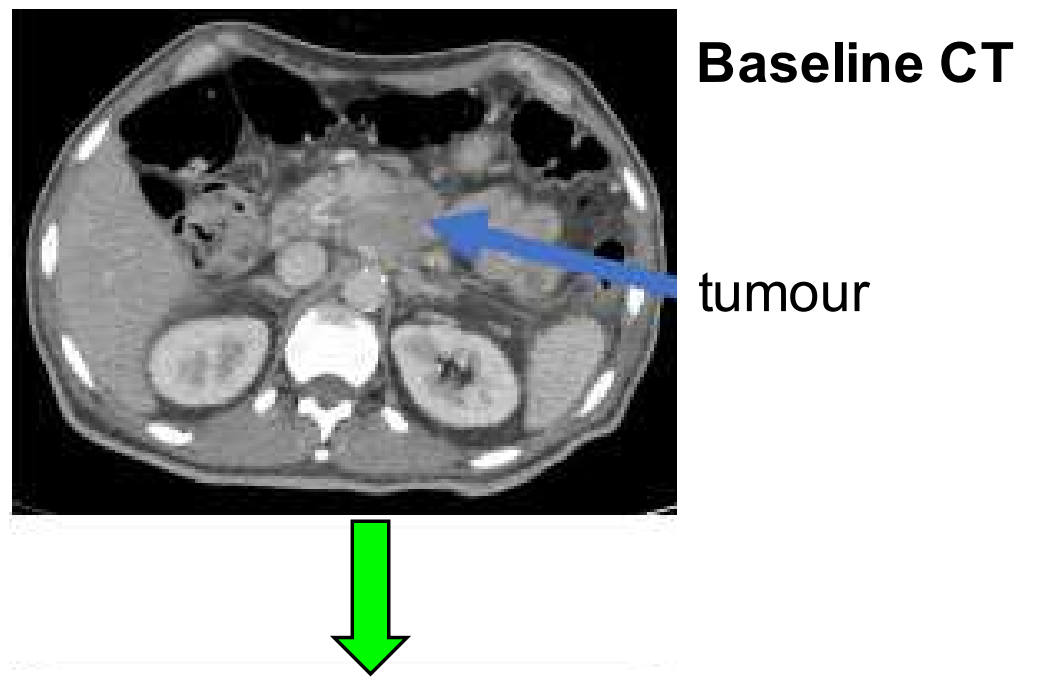

After 3x EUS RFA

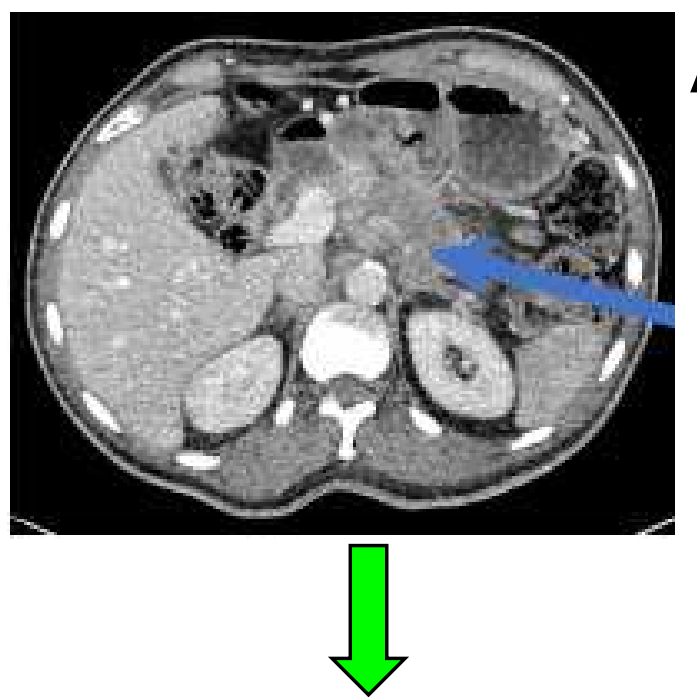

(Intervention)

Arm B

(Control)

EUS \& RFA on day 20 of each chemotherapy cycle; repeated for up to 3

consecutive cycles $\mathrm{N}=28$

Standard medical treatment $\mathrm{N}=28$

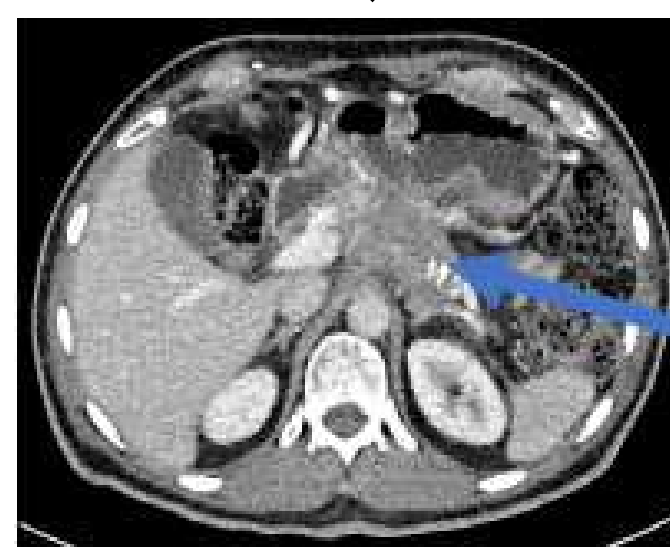

Primary outcome: Overall survival Secondary outcomes:

- Progression-free survival
16 months post treatment

tumour \& ablation site

- Radiological response \& disease control rate

- Assessment of quality of life

- Upregulation of immune system

- Safety (SAE occurrence) 


\section{Figure 2}

a. PDAC patients; $n=2$

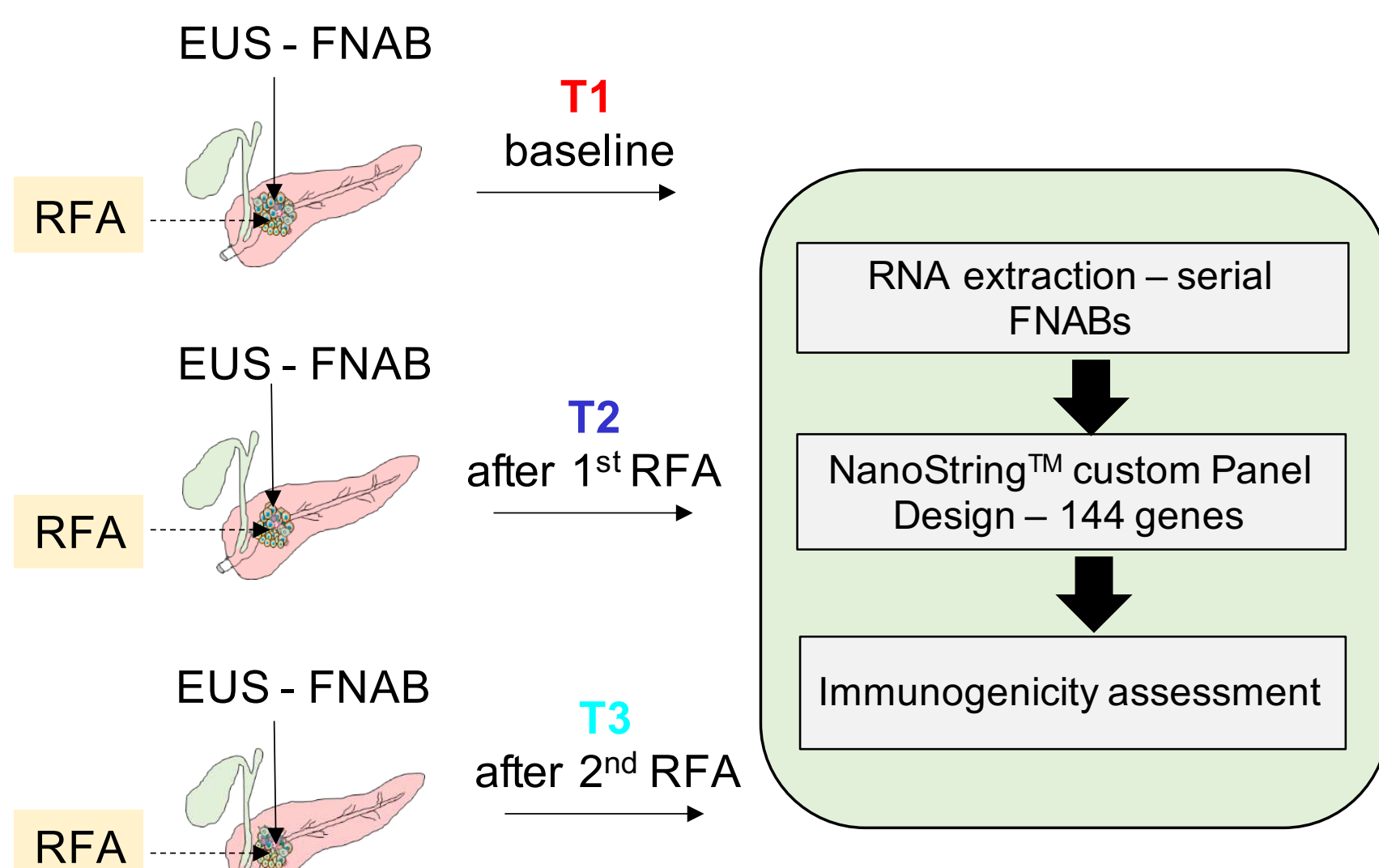

돌

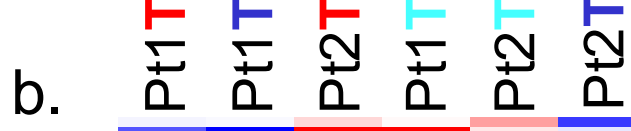
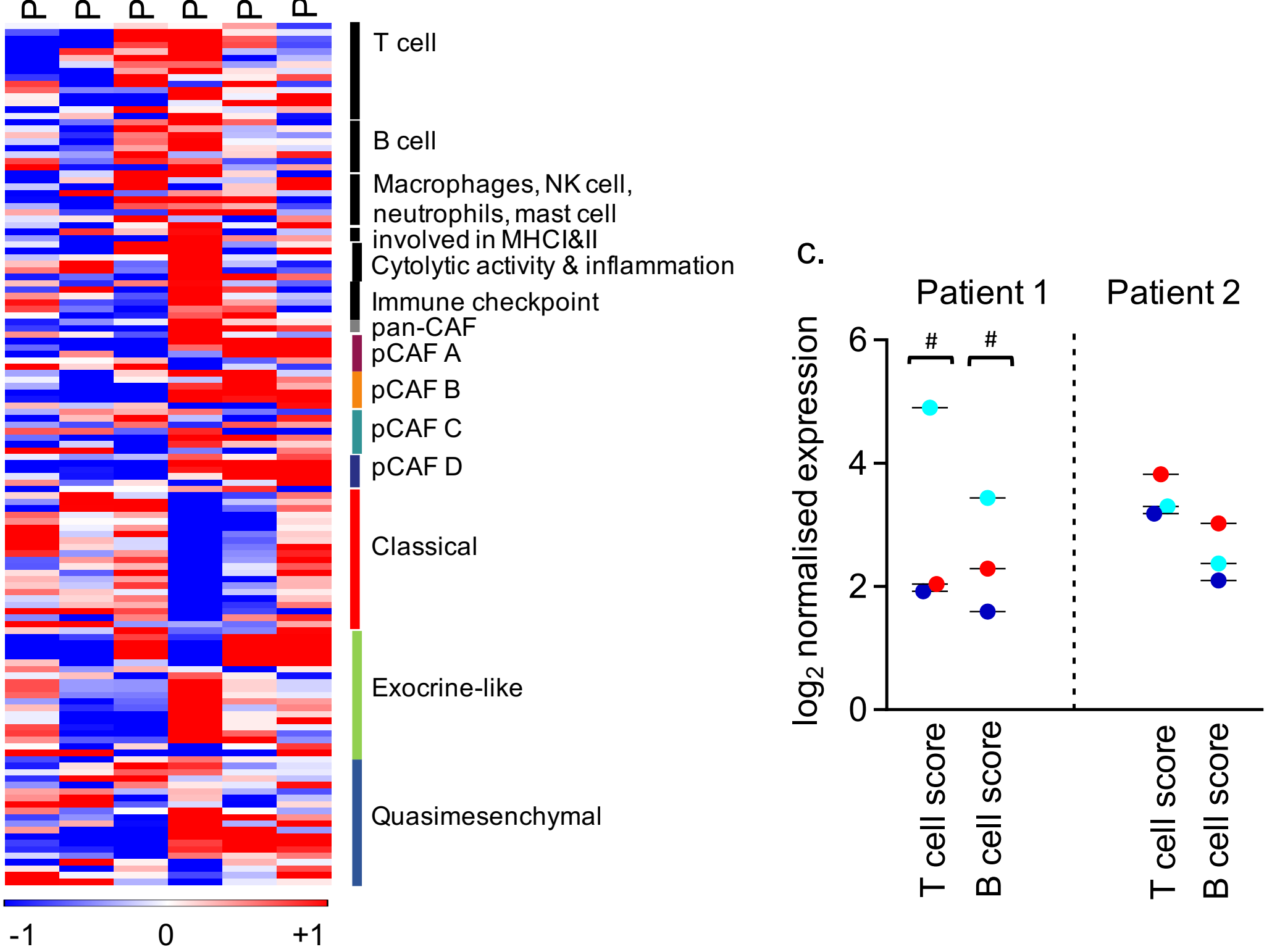



g.

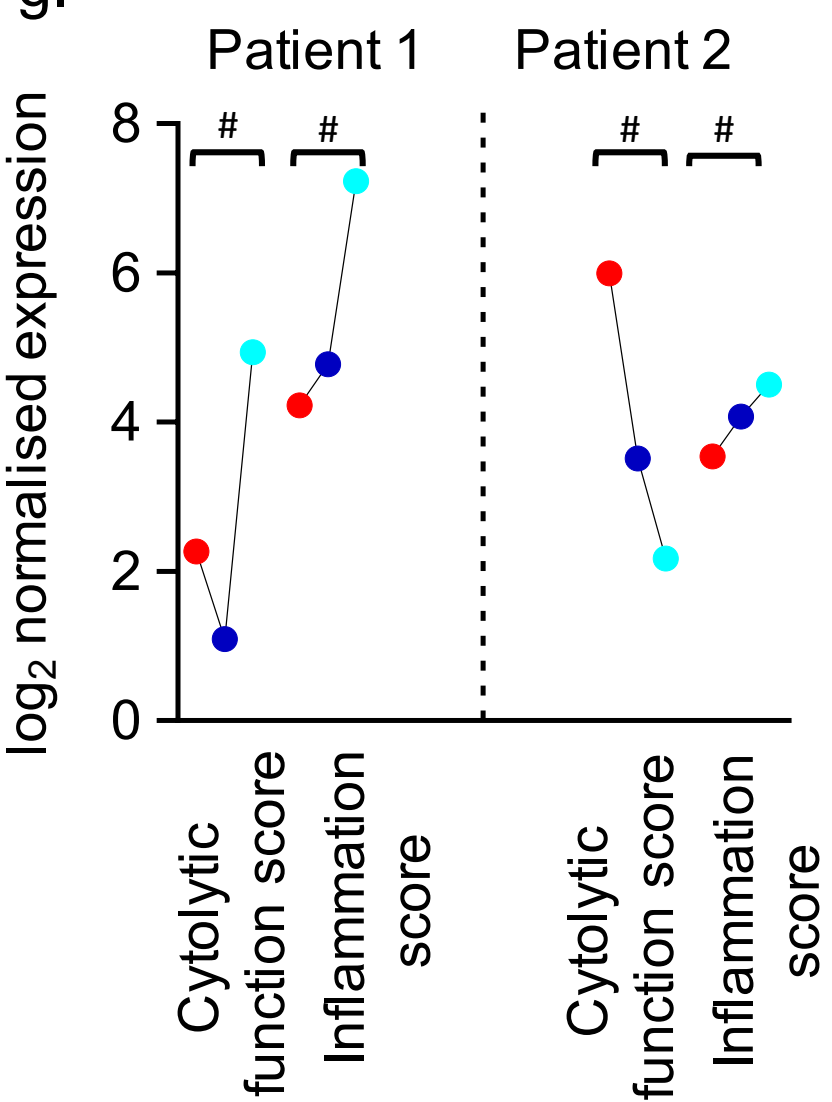

e.

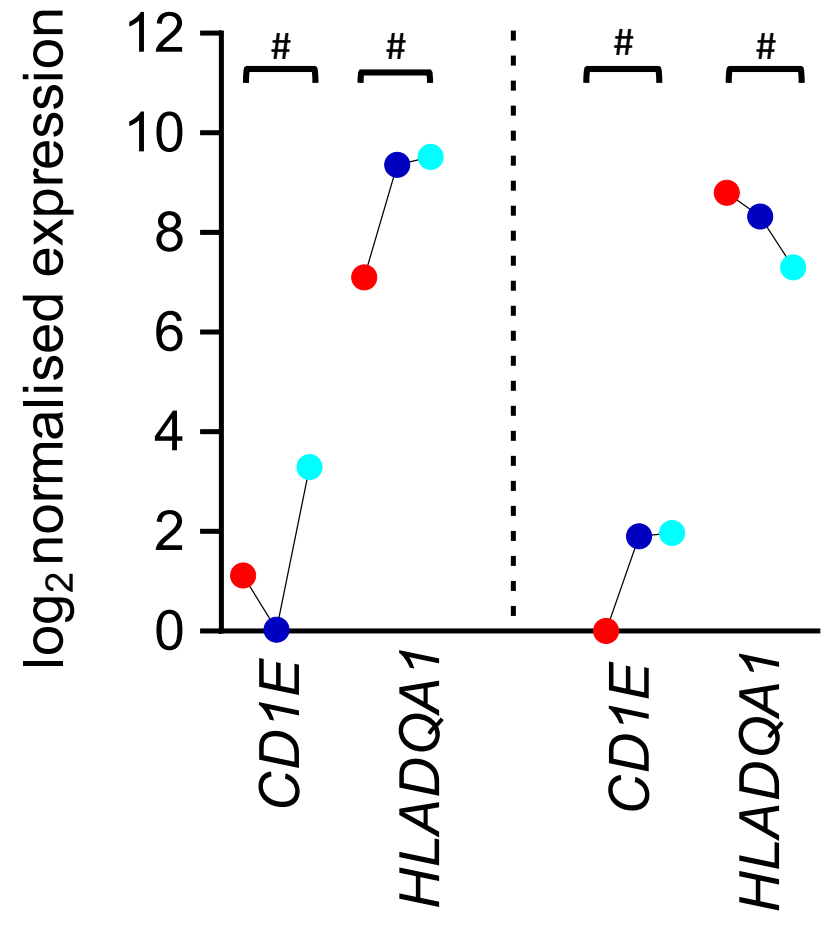

h

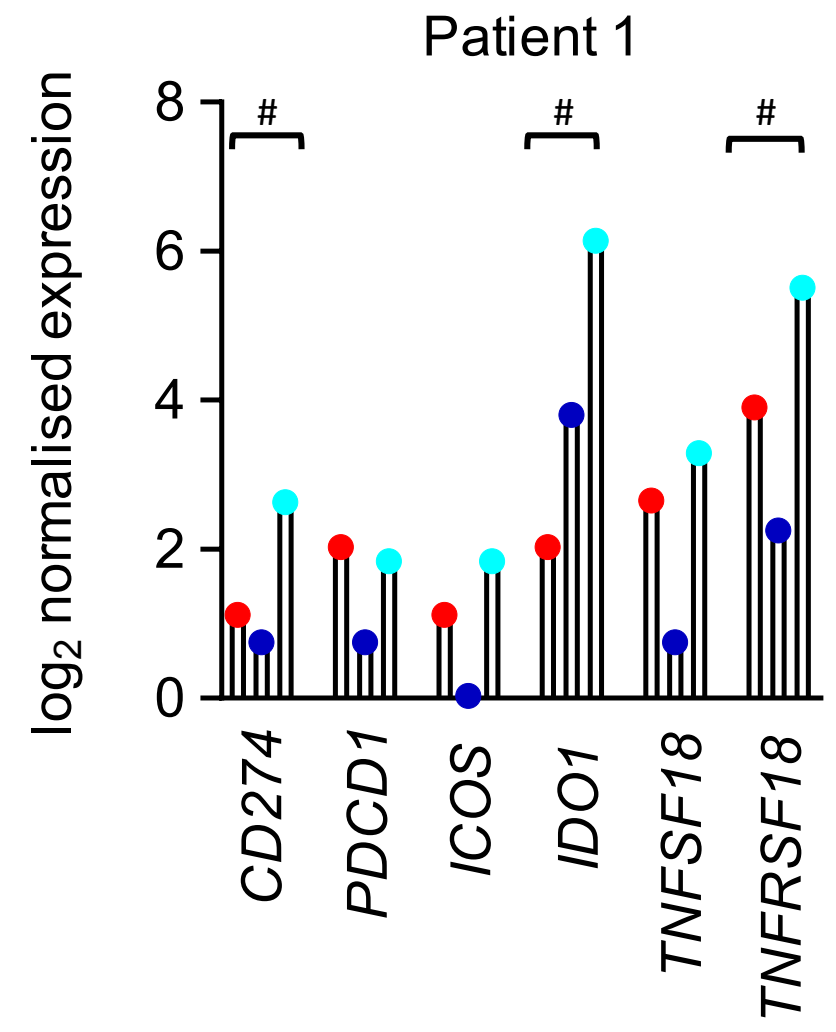

f. Immune cell types Patient 1


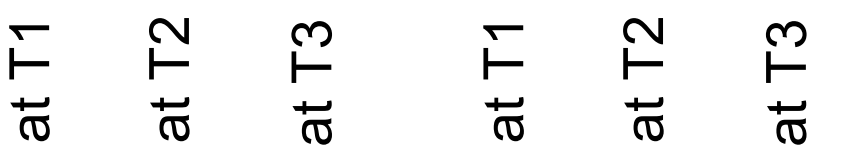

i.



Key

RFA treatment

- at T1

- at T2

- at T3

\# $\geq 2$-fold change 


\section{Supplementary Files}

This is a list of supplementary files associated with this preprint. Click to download.

- SupplementaryInformation.pdf 